\title{
A Systemic Review of the Auditory Training Program for Hearing Impairment
}

\author{
Joo Hong Kim, Sang Hee Lee, Eun Young Cho \\ Department of Special Education, Soonchunhyang University, Asan, Korea
}

\author{
Received: December 23, 2020 \\ Revised: February 3, 2021 \\ Accepted: March 4, 2021 \\ Correspondence: \\ Sang Hee Lee, PhD \\ Department of Special Education, \\ Soonchunhyang University, \\ 22 Soonchunhyang-ro, \\ Shinchang-myeon, \\ Asan 31538, Korea \\ Tel: $+82-41-530-1085$ \\ Fax: +82-41-530-1517 \\ E-mail: sanglh@sch.ac.kr
}

\begin{abstract}
The purpose of this study is to examine the current trend of research on auditory training programs for the hearing impaired through a systematic literature review, and to provide fundamental information in terms of program design and intervention of 24 studies conducted from 1998 to 2020 were selected according to the Participants, Intervention, Control, Outcomes, and Study design (PICOS) criteria. The quality of the study was evaluated, and then a systematic review of the literature was conducted. First, as a result of examining the selected auditory training program of hearing-impaired target, it was conducted in various experimental design methods including location, personnel, age, and intervention method. Second, as a result of examining different methods of intervention on independent variables, in case of auditory training programs, the most common intervention was conducted by using speech. For the assisting devices, the most common intervention programs were conducted by using speech with wearing hearing aid. Third, as a result of examining the intervention method on dependent variables, it was found that the most frequent intervention programs were conducted by using speech. For the further research, the auditory program for the hearing impaired shoud be proceeded at any place in family-friendly environment, and in the form of intervention implemented by caregivers through parental education. In addition, another intervention technique might be applied at untact interveniton using the internet and digital devices.
\end{abstract}

Key Words: Auditory program, Study quality, Systematic review.

\section{INTRODUCTION}

세계보건기구(World Health Organization, 2004)의 2004년 발표에 의하면 난청을 전 세계에서 열세 번째로 흔한 주요 장 애로 추정하고 있으며, 후천성 난청은 당뇨와 후천성 면역결핍 증후군의 발병 수를 넘어, 2030년에는 일곱 번째의 주요 장애 가 될 것이라고 보고하였다(Mathers et al., 2008). 우리나라의 경우, 2017년 기준 보건복지부에 등록된 인원만 302,003명이며 이 숫자는 고령화 사회로 됨에 따라 점점 심화될 전망이다.

보청기의 기술적 발전이 난청인의 장애 극복에 도움을 주지 만 소음 상황에서 의사소통을 개선하는 데 여전히 한계를 보이 고 있다. 이러한 난청인의 성공적인 청능재활을 제공하기 위해 서는 청능평가, 보장구 적합 및 상담과 함께 난청인 개개인에게 최적화된 청능훈련을 실시하는 것이 중요하다(Gordon-Salant, 2005; Lee \& Cho, 2011; Sweetow \& Sabes, 2006). 청능훈련

(c) This is an Open Access article distributed under the terms of the Creative Commons Attribution Non-Commercial License (https://creativecommons.org/licenses/by-nc/4.0) which permits unrestricted non-commercial use, distribution, and reproduction in any medium, provided the original work is properly cited.
은 난청인을 대상으로 청취 가능한 모든 음향적 단서를 최대한 활용할 수 있도록 지도하는 것으로, 난청인들이 잔존 청력(residual hearing)을 최대한 향상시켜 청력 손실로부터 발생될 수 있는 여러 가지 불이익을 최소화하고 궁극적으로는 일상생활 에서 보다 원활한 의사소통을 하도록 하는 것이다[American Speech-Language-Hearing Association (ASHA), 1984, 1996]. 청능훈련의 방법은 음소와 단어 위주(bottom-up approach)의 분석적(analytic), 청능훈련과 일상생활 속의 의사소통 방식과 유사한 문장, 이야기 등을 이용한 하향식 접근 방식(top-down approach)의 종합적(synthetic) 청능훈련으로 나눌 수 있다. 청 능훈련의 단계는 소리의 탐지(detection), 변별(discrimination), 확인(identification), 이해(comprehension)의 청각기술 발달 순 서를 기준으로 훈련에 사용할 자극음을 선택하여 실시한다. 예 를 들어, 선천적으로 난청의 경우, 청각적 어음을 학습하고, 청 각적 신호음과 어휘들의 연계성을 학습하는 데 목적이 있어 탐 지 수준부터 확인 또는 이해 수준까지 점차 난이도를 향상시키 는 훈련방법(Hong, 2013)을 사용한다. 이에 반해, 후천적으로 난청이 생기는 노인성 난청, 청력의 퇴화, 사고로 인한 난청과 
같이 선척적으로 듣기에 문제가 없었으나 어떠한 사건이나 시 간이 지남에 따라 난청이 생긴 경우는 인공와우나 보청기 등과 같은 보장구의 이득을 통해 듣기의 어려움을 완화시키므로 따 로 탐지나 변별 수준을 거치지 않고 보장구를 이용한 확인, 이 해 수준의 청능훈련(Cho et al., 2018; Lee \& Lee, 2017; Yeo et al., 2014)을 사용한다.

청능훈련 프로그램 관련 연구는 프로그램 개발 및 사례에 관한 연구가 주를 이루어왔다. 먼저 청능훈련 프로그램 개발에 관한 최근 선행연구들을 살펴보면 스마트폰 어플리케이션 활 용 및 실생활에서 나타나는 다양한 소재를 반영한(음식, 축제, 스포츠 등) 이야기 청능훈련 프로그램이 개발되었으며(Kim \& Bahng, 2017; Lee \& Lee, 2017; Yu et al., 2014), 청능훈련 사 례의 경우 대상, 장소, 중재방법 등 다양하게 이루어졌다(Cho et al., 2018; Hong, 2013; Jo et al., 2013; Kim \& Lee, 2017; Kim \& Lee, 2018; Kim \& Park, 2013; Lee et al., 2016; Yeo et al., 2014; Yoon et al., 2016). 또한 청능훈련에 참여하는 대 상의 특성이 다양한 만큼 연령과 착용보장구에 따른 중재방법, 다양한 현장에 적용기술에 따른 중재방법을 정량적인 근거 자 료를 바탕으로 현장의 교사 또는 청능사들에게 제공한다면, 객 관적이고 효과적인 훈련의 토대를 마련할 수 있을 것이다. 지금 까지의 선행연구들은 청능훈련 프로그램 효과를 알아보기 위 한 문헌분석들이 이루어졌는데, 시기적으로 최근의 이슈를 반 영하지 못하거나(Lee, 2005; Yoon \& Hwang, 2005), 청능훈련 프로그램의 동향 및 중재에 관하여 일반적인 분석으로(Park \& Han, 2019) 이루어진 것을 확인할 수 있었다. 더욱이 종래의 선 행연구들과 다르게 본 연구는 문헌의 질 평가를 실시하여 결과 분석을 도출하고, 체계적 문헌고찰을 살펴본 것이 의의가 있다.

이에 따라 본 연구는 청각장애대상 청능훈련 프로그램 연구 의 전반적인 동향을 체계적인 문헌 검색을 근거로 청능훈련 프 로그램의 현황, 중재방법, 효과 등을 살펴보고, 이를 바탕으로 청각장애대상 청능훈련 프로그램의 개발 및 시사점에 접근하 는 기초자료를 제공하고자 한다. 이를 위해 다음과 같은 연구 문제를 설정하였다.

첫째, 청각장애 대상의 청능훈련 프로그램 연구의 현황은 어 떠한가?

둘째, 독립변인(연령, 착용보장구)에 따라 청능훈련 프로그램
의 중재방법은 어떠한가?

셋째, 종속변인(의사소통, 생활기술, 의사소통 + 생활기술)에 따라 중재방법은 어떠한가?

\section{MATERIALS AND METHODS}

\section{데이터 추출}

본 연구는 청각장애 대상 청능훈련을 검증한 학술지 연구물 을 바탕으로 분석 대상을 선정하였으며 구체적인 분석기준은 다음과 같다.

첫째, 1998년부터 2020년까지 한국연구재단 등재 학술지에 게재된 연구 논문을 분석 대상으로 하였다. 둘째, 본 연구의 목 적에 따라 2020년 2월 8일부터 9월 15일까지 한국연구재단 (Korean Citation Index, KCI), 한국학술정보(Korean studies Information Service System, KISS), Korean Education and Research Information Service (KERIS; RISS), 교보문고 (KyoboScholar), 누리미디어(Nurimedia, DBpia), 국회전자도 서관, Korean Institute of Science and Technology Information [KISTI; National Digital Science Library (NDSL)]을 활 용하여 '청능훈련', '청능재활', '청각재활', '난청·효과', '청각장 애·효과를 검색어로 하여 대상 논문을 검색하였다. 그 외 적합 도가 높은 연구 논문의 참고문헌을 검색하여 취합하였다. 마지 막으로 PICOS에 따른 문헌 선정기준에 맞추어 유관기관 실시, 실험-통제집단, 사전-사후 실험설계, 통계적 결과 보고를 실시

Table 1. Criteria for selecting literature according to PICOS

\begin{tabular}{|c|c|}
\hline PICOS & Specific information \\
\hline Participants & Physically hearing handicapped person \\
\hline Intervention & Auditory training \\
\hline Comparison & Before/after auditory training \\
\hline Outcomes & $\begin{array}{l}\text { Speech recognition, listening satisfaction, } \\
\text { development, noise situation recognition }\end{array}$ \\
\hline Study designs & $\begin{array}{l}\text { Place, period (week), session (number of times), } \\
\text { session time (minute), retention period (month), } \\
\text { arbitration method (Program), } \\
\text { dependent variable, program results }\end{array}$ \\
\hline
\end{tabular}

PICOS: Participants, Intervention, Control, Outcomes, and Study design

Table 2. Inclusion and exclusion criteria for literature review used in the study

Type Inclusion Exclusion

Paper publication year From 1998 to September 2020

Before 1998, after September 2020

Types of literature Korean Research Foundation registered literature

Study design Program development and experimental study

Foreign literature, unregistered literature

Study design

Auditory training, auditory rehabilitation,

Meta-analysis and systematic analysis, Essay (article)

Excluded search terms

hearing rehabilitation, hearing loss \& effect, deafness 
한 논문을 분석 대상으로 선정하였다. 본 연구에서 사용된 PICOS에 따른 문헌 선정기준은 Table 1 , 본 연구의 문헌분석 에 적용된 기준은 Table 2 와 같다.

체계적분석 및 메타분석에서 기본적인 보고 항목으로(Moher et al., 2009), 연구의 과정을 나타내는 Preferred Reporting Items for Systematic Reviews and Meta-Analyses (PRISMA) 플로우차트(flowchart)를 제시하면 Figure 1과 같다.

청각장애 대상 청능훈련 프로그램 연구 동향을 검증한 연구 물 중 1 차로 취합된 연구물은 학술지는 50 편으로 나타났다. 문 헌 선별 및 선정과정에서 초록 검토를 통해 본 연구와 적합도 가 낮은 연구 20편을 제외하였다. 이후 30편의 full text 점검을 통해 학위와 학술지 중복연구, 부정확 표기, 청각장애 대상이 아닌 연구물 등 본 연구의 $\mathrm{PICOS}$ 기준에 부적합하다고 판단 된 문헌은 최종 선정 문헌에서 제외하였다.

\section{신뢰도 확인}

체계적 문헌고찰 및 메타분석에서 자료추출 인원과 포괄적 인 문헌검색, 분석 결과에 대한 최종 합의 기준을 제시한 The Assessment of Multiple Systematic Reviews (AMSTAR)에 따르면, 문헌을 선정하는 과정에서 연구자의 주관성을 방지하 고 명확한 기준과 평가의 신뢰성을 확보하기 위해서 2 인 이상 이 자료추출과 코딩에 참여하는 것을 매우 중시한다(Seo \& Moon, 2017; Shea et al., 2007). 이에 본 연구는 청각장애전공 교수 1 인, 교육심리학 전공 박사 1 인, 청각재활 전공 석사 1 인에 의해 full text 점검 논문의 $100 \%$ 를 독립적으로 분석하였고, 코 딩이 일치하는 세부 관련변수/(코딩이 일치하는 세부 관련 변 수 + 일치하지 않는 세부 관련변수) $\times 100$ 으로 산출한 결과 (Jung \& Choi, 2019) 평정자간 신뢰도는 92.85\%로 나타났다.

\section{최종문헌 선정 및 분류기준}

청각장애 대상 청능재활 프로그램의 체계적 문헌분석을 위 해 최종 선정한 문헌은 학술지 24편이며 청각장애 대상 청능훈 련 프로그램의 효과 분석을 위해 Cooper(2010)가 제시한 내용 을 바탕으로 Byun et al.(2017), Cho \& Lee(2019), Lee \& Jeon (2013)이 재구성하여 사용한 분석틀을 적용했다. 이에 저자, 출 판연도, 대상 수, 나이, 착용 보장구, 장소, 회기 수, 기간, 회기 시 간, 유지기간 여부, 상담 여부, 중재방법, 종속변인을 분석하였 고 독립변인은 나이, 착용 보장구에 따른 교수방법으로 분류하 였다. 첫째, 교수방법은 청각장애 전공 교수 1 인, 교육심리학 전 공 박사 1 인, 청각재활 전공 석사 1 인에 의해 각 연구물에 연구 자가 기술한 교수방법 이외, 제시된 중재계획안을 분석하여 분 석자 간 일치하는 교수방법을 채택하였고, 이에 이의가 있는 연 구물의 경우 저자에게 확인하는 과정을 거쳤다. 그 결과를 바 탕으로 어음, 환경음, 소음, 스마트기기, 음악에 따라 분류하여 분석하였다. 둘째, 연구방법은 대상 수, 연령, 착용보장구에 따 른 독립변인의 적용에 따라 분석하였다. 마지막으로 종속변인 은 의사소통, 생활기술(청능훈련, 보청기, 소음 상황에서 듣기 만족도 등, 청각 기억력, 주의집중, 듣기능력에 영향을 줄 수 있 는 기술) 의사소통 + 생활기술의 세 가지 범주로 분류하였으며 상세내용은 Table 3과 같다.

\section{질적 평가}

체계적 문헌고찰을 위해 포함된 문헌들의 질(quality) 평가는 비뚤림이 최소화(내적 타당도의 정도)된 결과를 반영하여 의미 있는 고찰을 도출하게 하고, 추정치를 과대 혹은 과소 추정을 막기 위해 필수적으로 수행해야 한다(Lee \& Choi, 2012). 이에 Park \& Han(2019)에서 적용한 Study validity criteria, study

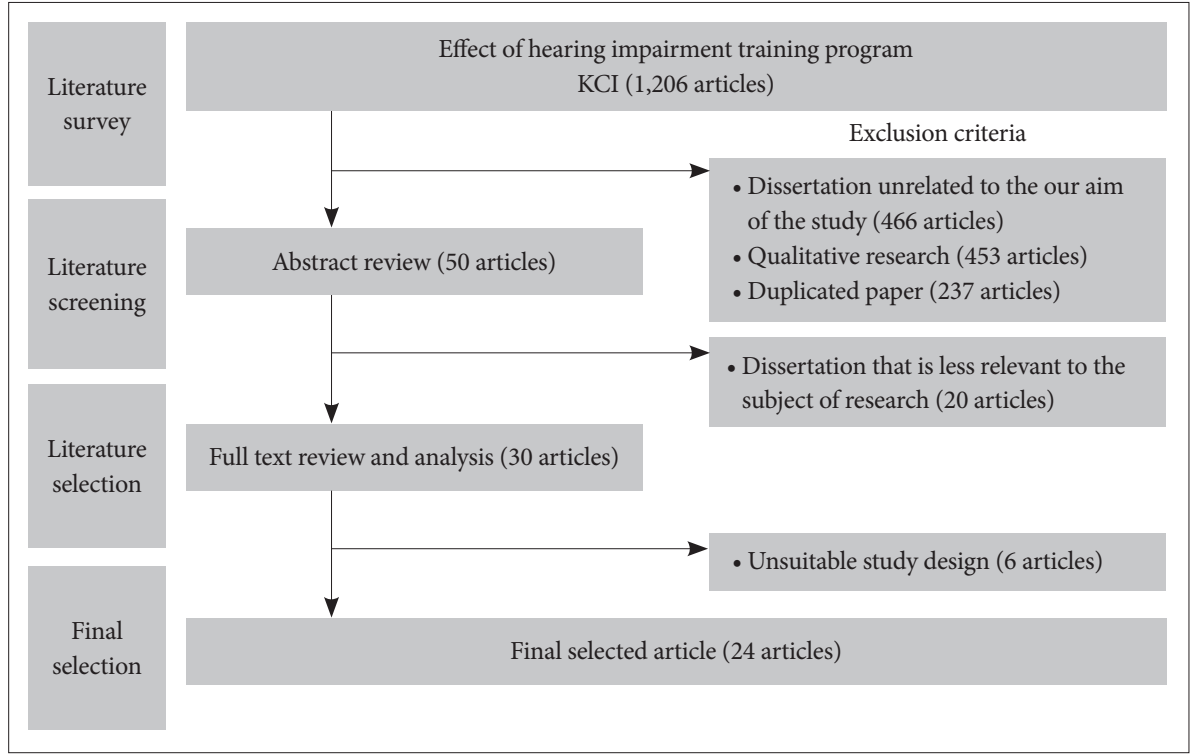

Figure 1. A flow chart of PRISMA. PRIS$M A$ : Preferred reporting items for systematic reviews and meta-analyses, $\mathrm{KCl}$ : Korea Citation Index. 
Table 3. Standard for classification of arbitration methods and dependent variables

\begin{tabular}{ll}
\hline \multicolumn{1}{c}{ Type } & \multicolumn{1}{c}{ Specific information } \\
\hline Teaching method & High frequency word, vocabulary, phono recognition \\
Speech & Environmental sound \\
Environmental sound & Noise situation auditory training \\
Noise situation recognition & Video, smart device, smartphone \\
Smart device & Music, choral \\
Music & Check consonants, vowels, word recognition, sentence recognition, phono recognition \\
Dependent variable & Noise situation speech recognition, subjective satisfaction, attention, memory \\
Communication & Communication and living skill duplicate \\
Living skill &
\end{tabular}

quality scores and levels of evidence for included articles를 참조하여 10개의 문항(Randomization, Control group, Power calculation, Blinding, Outcome measure reporting, Outcome measure selection, Training feedback, Ecological validity, Reporting of compliance, Follow-up)을 기반으로 24개의 문 헌에 대하여 평가를 실시하였다. 질적 평가는 0 2점으로 측정 하였다. 0 점은 잘못되었거나 판단을 할 수 있는 정보가 없음을 나타내며, 1 점은 적은 정보를 포함하고 있거나 구체적인 정보가 없음을 의미하고, 2점은 구체적인 정보의 적절한 사용 및 보고 를 의미한다. 각 문헌의 개별 척도 점수를 합산하여 $0 ~ 5$ 는 매 우 낮음, 6 10은 낮음, 11 15는 보통, 16 20은 높음으로 평가하 였다. 평가는 특수교육학과 교수 2 인, 교육학박사 1 인에 의해 실 시되었으며, 비뚤림 위험 평가 도구인 Cochrane 그룹에서 제공 하는 Revman을 이용하여 나타낸 결과는 Figure 2 와 같다.

24건의 문헌의 질적 평가를 진행한 결과 평균 $9.00( \pm 2.73)$ 점 으로 전반적으로 낮음(low) 수준이었다. 18건의 문헌이 낮음 (low) 수준이었으며, 중간(moderate) 수준의 문헌은 총 5건, 높 음(high) 수준의 문헌은 총 1건이었다. 문헌의 질 평가를 통해 무작위 배정순서 생성 및 은폐, 연구참여자, 연구자 및 결과평 가에 대한 눈가림, 교란 변수 등에 해당하는 부분이 불확실하 게 기술된 문헌이 일부 포함된 것으로 확인되었으나, 본 연구를 통해 청능훈련 프로그램의 연구 현황을 살펴보고 연구결과들 을 종합하여 제시하고자 한다는 측면을 고려한 Kim et al. (2018)과 같이 모든 연구를 분석 대상에 포함하였고, 자세한 분석 논문은 Table 4 및 Appendix에 제시되어 있다.

\section{RESULTS}

\section{연구 데이터}

\section{대상자}

청능훈련 프로그램의 대상 수는 1명(Cho et al., 2018; Hong,
2013; Jo et al., 2013; Kim \& Lee, 2018; Lee \& Cho, 2011; Yoon et al., 2016; Yu et al., 2014)부터 20명(Lee, 2018)으로 평 균 5 명으로 나타났다. 대상 연령은 각 문헌별 평균연령을 본 결 과, 9개월(Yoon et al., 2016)부터 79세(Lee \& Bahng, 2020)이 며 평균 25세로 나타났다. 대상자는 전부 보장구를 착용하였으 며 보장구 착용 정도는 보청기, 인공와우, 보청기 + 인공와우 순으로 많이 착용한 것으로 나타났다.

\section{연구설계}

장소는 장소를 기입하지 않은 문헌 4건(Hwang \& Seok, 2007; Lee \& Cho, 2011; Park et al., 2019; Tark et al., 2019)을 제외 하고 살펴본 결과, 방음실, 치료실, 가정, 조용한 방, 센터, 강의 실, 교실, 집 등으로 다양하였다.

회기 수는 5 회(Lee \& Lee, 2017)부터 90회(Kim \& Seok, 1998)이며 평균 19.48회, 표준편차 18.39로 나타났고 기간은 4 주(Cho et al., 2018; Yu et al., 2014)부터 36주(Lee, 2018)로 평 균 11.36 주, 표준편차 6.84로 나타났다. 회기 수와 기간의 경우 중복 데이터가 존재하는 문헌(Kim \& Seok, 1998)으로 인하여 전체 대상을 25 개로 설정하였다. 훈련시간은 10 분(Choi \& Park, 2019)부터 120분(Lee, 2018)으로 평균 44.31분, 표준편차 23.00으로 나타났다.

유지기간을 둔 문헌은 8건(Hwang \& Seok, 2007; Kim \& Park, 2013; Kim \& Seok, 1998; Lee et al., 2016; Lee et al., 2019; Lee \& Choi, 2017; Tark et al., 2019; Yoon et al., 2016) 으로 최종선정 문헌 중 33.33\%에 해당하며, 상담시간을 표기 한 문헌은 8건(Cho et al., 2018; Kim \& Lee, 2017; Kim \& Lee, 2018; Lee et al., 2019; Lee \& Bahng, 2020; Lee \& Lee, 2017; Yeo et al., 2014; Yoon et al., 2016)으로 최종 선정 문헌 에 $33.33 \%$ 에 해당하였다. 실험군과 대조군을 비교한 문헌은 5 건(Kim \& Bahng, 2017; Kim \& Lee, 2017; Lee \& Bahng, 2020; Tark et al., 2019; Yeo et al., 2014)이며 최종선정 문헌 중 $20.83 \%$ 에 해당하였다. 


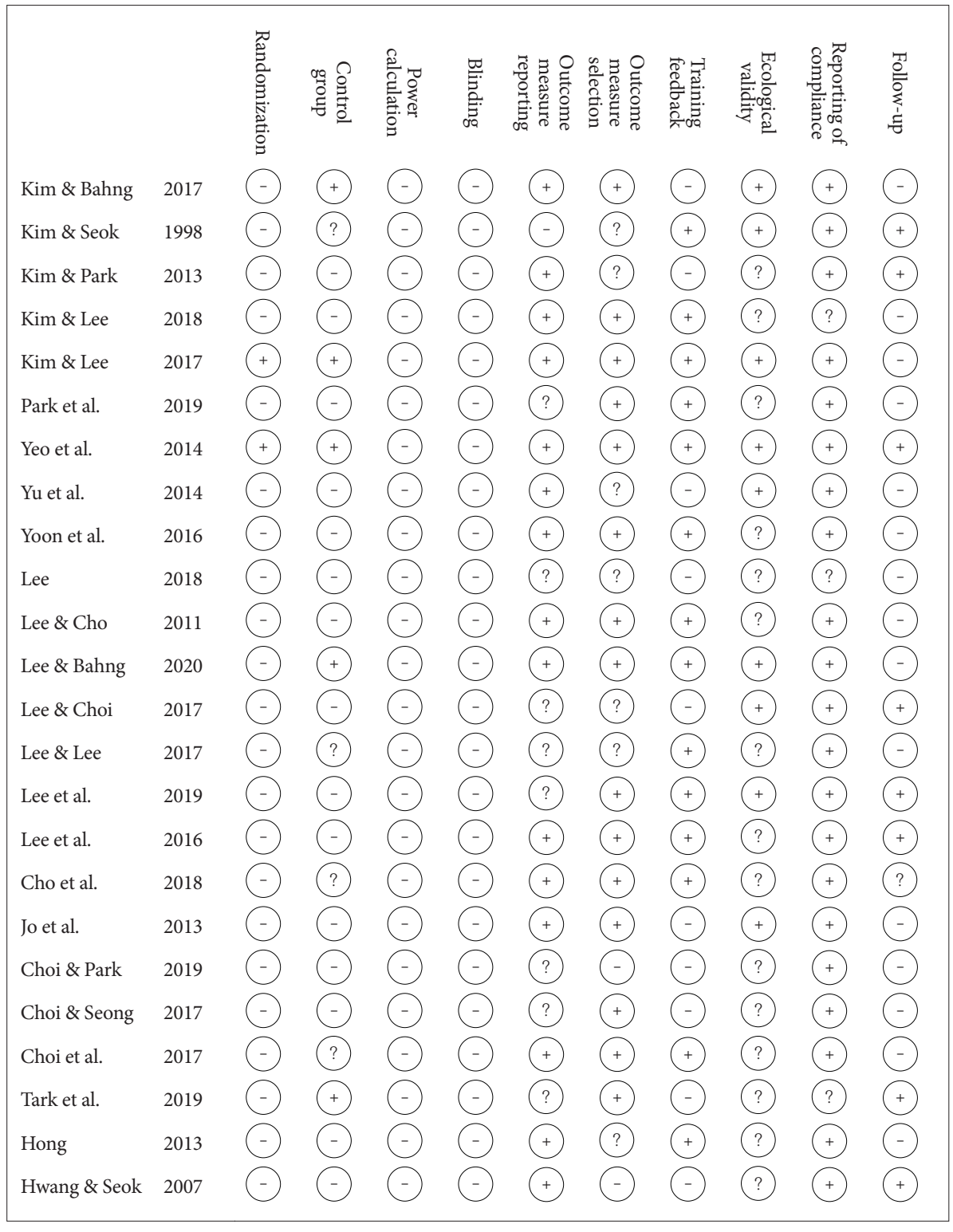

Figure 2. The individual sclae score of literature risk of bias ('-' means that the information related to auditory training is inaccurate or there is no information that can be judged. '?' means that the there is little or no specific information, and ' + ' means the use and reporting of specific information).

\section{독립변인}

중재방법은 연구자마다 다양한 방식의 청능훈련 프로그램을 고안해 사용하였다. 전반적인 중재방법의 세부요인을 살펴보면 다음과 같다. 이야기를 통한 청능훈련 2건(Cho et al., 2018; Kim \& Bahng, 2017), 청각을 통한 언어재활 1건(Kim \& Seok, 1998), 어휘지도 2건(Hong, 2013; Kim \& Park, 2013), 환경음과 이야기를 이용한 청능훈련 1건(Kim \& Lee, 2018), 환경음을 이 용한 청능훈련 2건(Kim \& Lee, 2017; Lee et al., 2016), 무의미

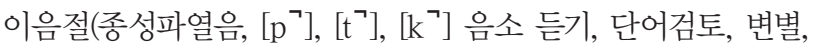
확인) 최소대조짝을 통한 청능훈련 1건(Park et al., 2019), 소음 하 청능훈련 2건(Lee et al., 2019; Yeo et al., 2014), 스마트 기 기 2건(Lee \& Lee, 2017; Yu et al., 2014), Korean aural rehabilitation for infants (KARI)를 이용한 조기재활 1건(Yoon et al., 2016), 중급 합창프로그램 1건(Lee, 2018), 탐지, 변별 훈련 1 건(Lee \& Cho, 2011), 동영상 1건(Lee \& Bahng, 2020), 음운인 식 1건(Lee \& Choi, 2017), 시간압축 문장 1건(Jo et al., 2013), 마음읽기(얼굴표정 사진 표현, 같은 정서 사진 짝짓기) 중재 1건 (Choi \& Park, 2019), 컴퓨터기반 듣기 프로그램 1건(Choi \& Seong, 2017), 음악을 이용한 청능훈련 2건(Choi et al., 2017; Tark et al., 2019), 차이전략(참조물과 비참조물들을 구별될 수 있도록 강조하기)을 강화한 장벽게임 1건(Hwang \& Seok, 2007)이었다.

\section{종속변인}

청각장애대상 청능훈련 프로그램은 연구자마다 다양한 방식 을 사용하였고 이에 따른 종속변인 또한 다양하게 나타났다. 


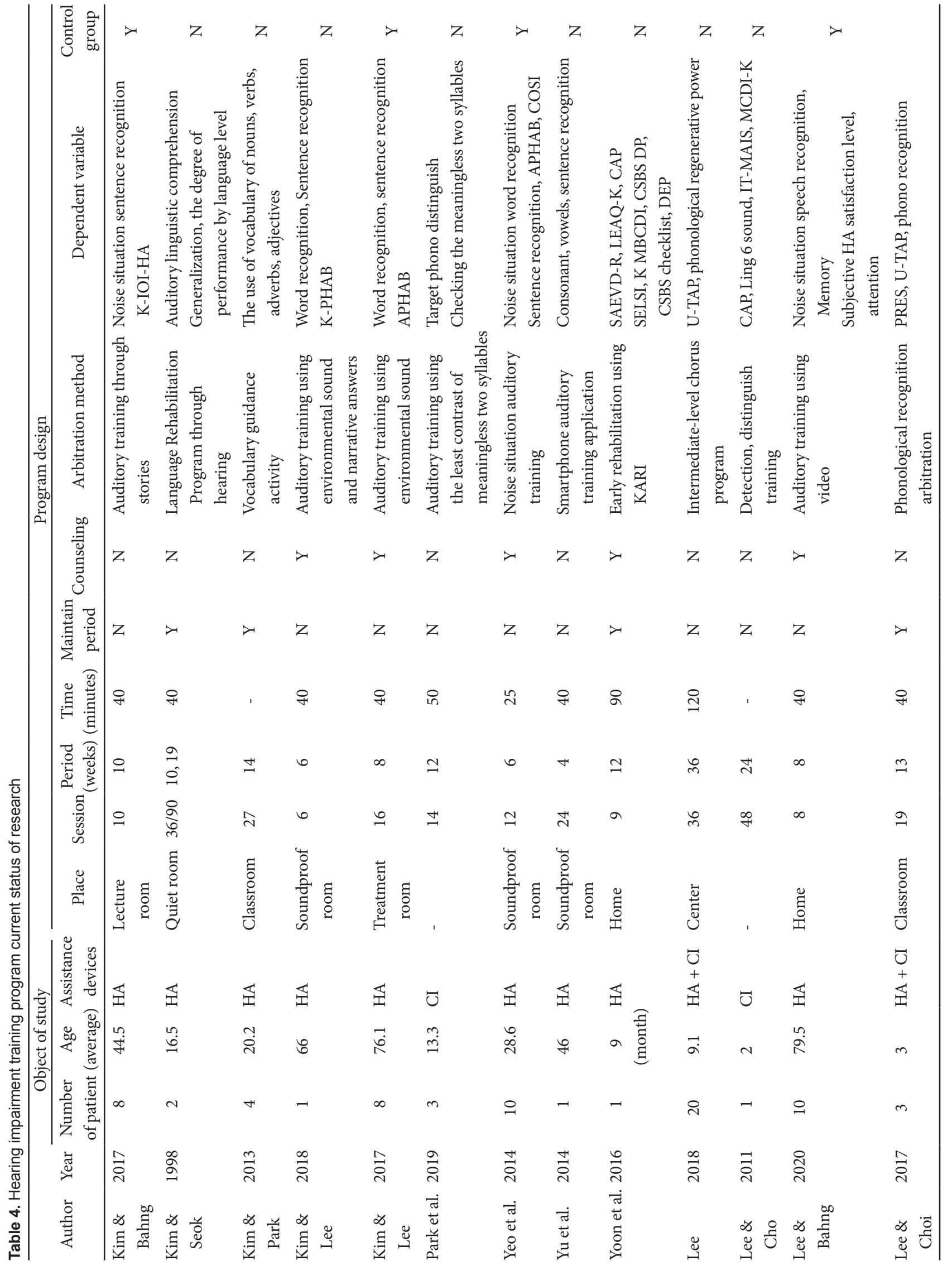


Systemic Review Study on the Auditory Training Program

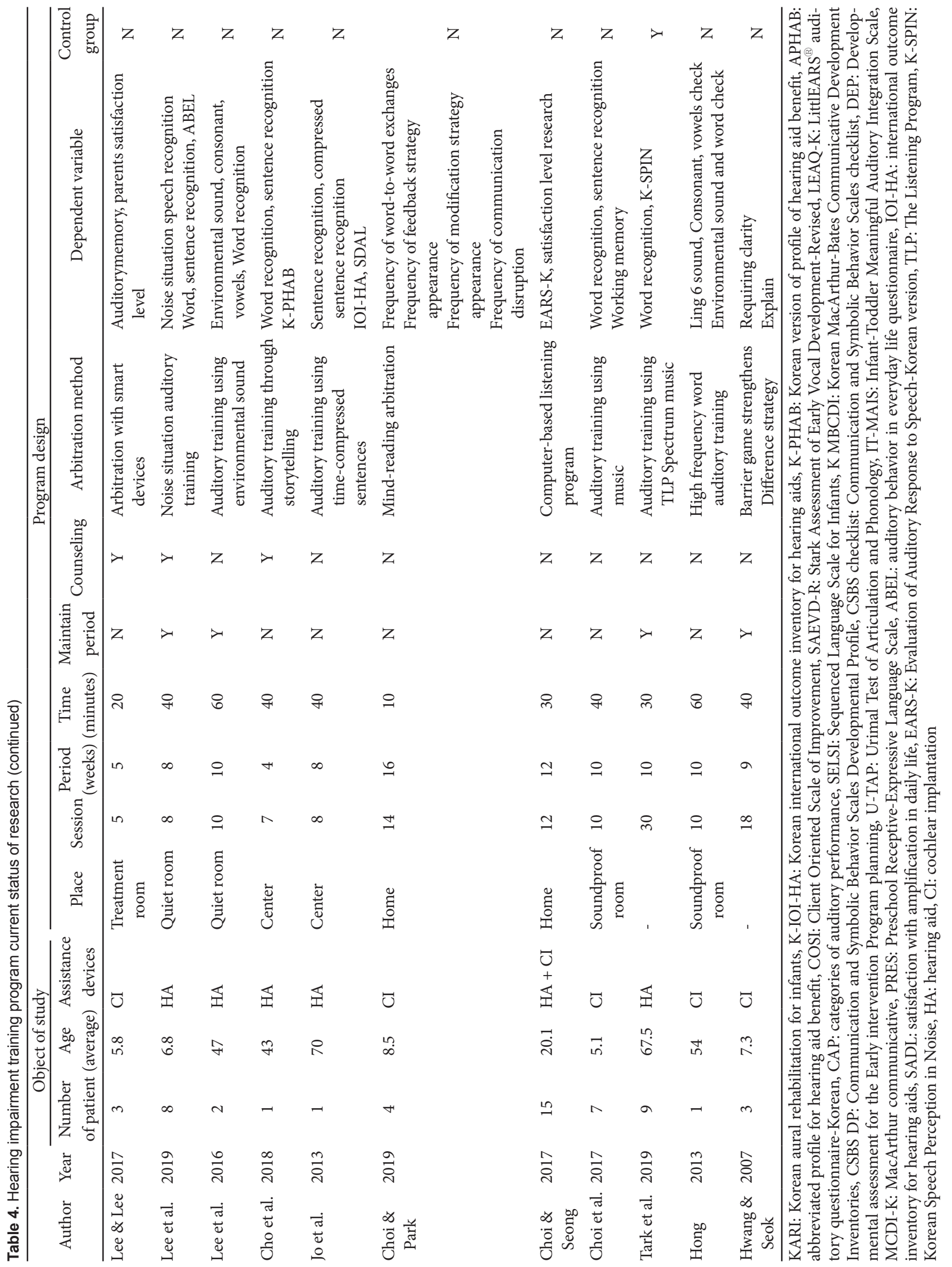


종속변인의 예를 살펴보면 다음과 같다. 소음하 단어, 문장, 어 음 인지도(Kim \& Bahng, 2017; Lee, et al., 2019; Lee \& Bahng, 2020; Tark, et al., 2019; Yeo et al., 2014), Ling 6 sound(Hong, 2013; Lee \& Cho, 2011), 자음, 모음, 단어, 문장, 어 음 인지도(Cho et al., 2018; Choi et al., 2017; Choi \& Seong, 2017; Jo et al., 2013; Kim \& Lee, 2017; Kim \& Lee, 2018; Lee et al., 2016; Lee et al., 2019; Yeo et al., 2014; Yu et al., 2014; Tark et al., 2019), 압축 문장인지도(Jo et al., 2013), 부모 또는 주관적 만족도(Cho et al., 2018; Choi \& Seong, 2017; Jo et al., 2013; Kim \& Bahng, 2017; Kim \& Lee, 2017; Kim \& Lee, 2018; Lee \& Bahng, 2020; Lee \& Lee, 2017; Yeo et al., 2014), 기억력, 작업기억력, 청각기억력(Choi et al., 2017; Lee \& Bahng, 2020; Lee \& Lee, 2017) 음소변별(Park et al., 2019), 음소, 음절, 단어 확인(Hong, 2013; Park et al., 2019), 의사소 통능력(Choi \& Park, 2019; Hwang \& Seok, 2007; Kim \& Seok, 1998), 음고 재생력(Lee, 2018), 주의력(Lee \& Bahng, 2020), 조음능력(Lee, 2018; Lee \& Choi, 2017), 언어 및 어휘 발 달(Kim \& Park, 2013; Lee \& Choi, 2017; Yoon et al., 2016), 음운 인식(Lee \& Choi, 2017), 청각적 반응능력 설문지 InfantToddler Meaningful Integration Scale (Lee \& Cho, 2011), 주 변환경 소리 인지능력 설문지(Categories of Auditory Performance; Lee \& Cho, 2011), 일상생활 청각행동 설문지(Auditory Behavior in Everyday Life questionnaire; Lee et al., 2019)가 있었다. 이상 24 개의 문헌에서 추출한 데이터 PICOS 기준에 따라 제시하였고 문헌에서 연구의 질적평가를 위해 추 출한 데이터 자료는 Table 4와 같다.

\section{독립변인에 따른 청능훈련 프로그램 중재방법}

독립변인에 따른 청능훈련 프로그램의 교수방법은 연구자마 다 다양하게 구안하여 사용하고, 한 가지 요인으로 동일하게 중재프로그램을 사용하는 것은 어려움을 보였다. 따라서 독립 변인에 따른 교수방법은 어음, 환경음, 소음하 인지, 스마트기 기, 음악으로 범주화하여 살펴본 결과, 어음이 13 건으로 가장 많았고 이는 $54.16 \%$ 의 비중을 차지하였다. 그 다음으로는 스마 트기기, 음악 순이었고 가장 적은 중재방법은 환경음과 소음하 인지로 각각 2 건으로 $8.33 \%$ 에 해당하는 것으로 나타났다. 이 를 연령과 착용보장구로 구별하여 살펴본 청능훈련 프로그램 의 교수방법 결과는 Table 5 와 같다.

연령과 상관없이 모든 나이대에서 어음이 가장 많이 사용되 었으며, 세부적으로 살펴보면 다음과 같다. 3세 미만의 경우, 어 음을 이용한 중재가 사용되었고(Lee \& Cho, 2011; Yoon et al., 2016), 3 7세의 경우, 어음이 가장 많았으며 소음하 인지와 스마트기기, 음악이 각각 1번씩 사용되었다. 8 19세의 경우, 어
음과 음악을 이용한 중재가 사용되었고 20 64세의 경우, 음악 을 제외한 모든 중재가 사용되었다. 65 세 이상 노인의 경우는 소음하 인지를 제외한 모든 교수 방법이 사용되었다.

착용 보장구에 따른 청능훈련 프로그램 중재방법은 다음과 같다. 보청기 착용 어음을 사용한 중재프로그램 $29.16 \%$, 인공 와우 착용 어음을 사용한 중재프로그램 $20.83 \%$, 보청기+인공 와우 착용 어음 및 스마트기기, 음악을 사용한 중재프로그램 $4.16 \%$ 순으로 나타났고, 환경음과 소음하 인지 중재프로그램 은 사용되지 않았다.

\section{종속변인에 따른 청능훈련 프로그램 중재방법}

종속변인에 따른 청능훈련 프로그램의 교수방법은 연구마다 청능훈련 프로그램이 다르고 그에 따른 사전/사후 검사가 다르 며 보고자 하는 결과값이 다르게 나타났다. 종속변인에 따른 청능훈련 프로그램의 교수방법인 의사소통 언어, 사회적 생활 기술, 의사소통 언어+사회적 생활기술로 범주화하여 살펴본 결 과는 Table 6과 같다.

의사소통 범주에서는 어음을 사용한 중재프로그램 $20.83 \%$, 환경음을 사용한 중재프로그램 $4.16 \%$, 스마트기기를 사용한 중재프로그램 $4.16 \%$ 순으로 나타났다. 그리고 생활기술 범주에 서는 어음을 사용한 중재프로그램 $20.83 \%$, 스마트기기 $8.33 \%$, 환경음 및 소음하 인지, 음악을 사용한 중재프로그램은 나타나 지 않았다. 또한 의사소통 + 생활기술을 사용한 중재프로그램 은 어음 $12.5 \%$, 음악 $12.5 \%$, 소음하 인지 $8.33 \%$, 스마트기기 $4.16 \%$, 소음하 인지 $4.16 \%$ 순으로 나타났다.

\section{DISCUSSIONS}

본 연구는 청각장애대상 청능훈련 프로그램 현황, 중재방법, 효과 등 연구의 전반적인 동향을 체계적인 문헌고찰 방법을 사 용하여 분석하였으며, 이를 통해 청각장애대상 청능훈련 프로 그램의 개발 및 시사점에 접근하는 자료를 제공하는 데 목적이 있다. 이를 위해 최종 선정된 24편의 문헌은 청각장애 대상 청 능훈련 프로그램 효과분석을 위하여 프로그램 목표와 독립변 인 및 종속변인에 따른 중재방법을 살펴보았으며, 결과에 따른 논의는 다음과 같다.

첫째, 청각장애 대상의 청능훈련 프로그램 연구의 대상선정 (거주지, 대상자 수, 착용 보장구, 연령) 기준으로 살펴본 결과 보장구의 경우 보청기를 대상으로 한 연구가 가장 많았다(14 편, 58.33\%). 연구 대상자의 연령은 기존에 보장구를 착용하고 있었던 노년층을 대상으로 한 연구보다는 신규 또는 중장년층 을 대상으로 한 연구가 다수인 것으로 보아 보장구 중에서 수 요가 많은 보청기와 점차 낮아지는 대상자의 착용연령에 대해 
Table 5. Method of arbitration of hearing training programs according to independent variables

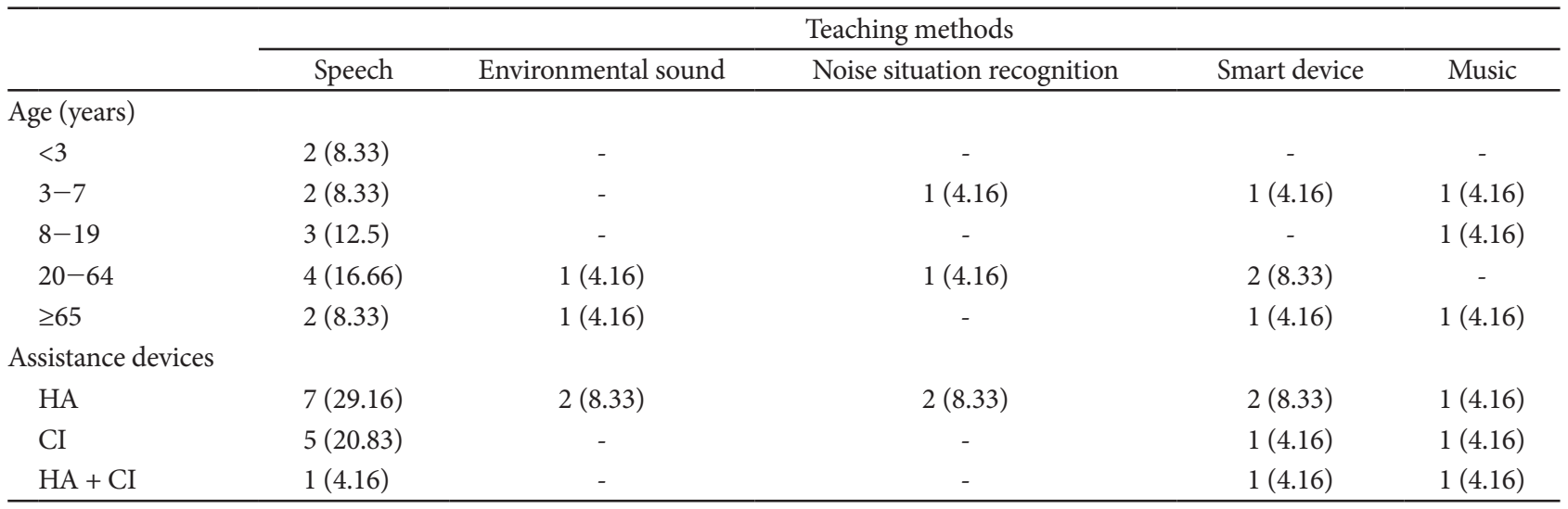

Values are presented as n (\%). HA: hearing aid, CI: cochlear implant

Table 6. Method of arbitration of hearing training programs according to dependent variables

\begin{tabular}{llcccc}
\hline \multirow{2}{*}{ Dependent variable } & \multicolumn{4}{c}{ Teaching methods } \\
\cline { 2 - 6 } & Speech & Environmental sound & Noise situation recognition & Smart device & Music \\
\hline Communication & $5(20.83)$ & $1(4.16)$ & - & $1(4.16)$ & - \\
Living skill & $5(20.83)$ & - & - & $2(8.33)$ & - \\
Communication + Living skill & $3(12.5)$ & $1(4.16)$ & $2(8.33)$ & $1(4.16)$ & $3(12.5)$ \\
\hline
\end{tabular}

Values are presented as $\mathrm{n}(\%)$.

연구의 초점이 맞춰지고 있다는 것을 알 수 있다.

둘째, 청각장애 대상의 청능훈련 프로그램 향상을 위한 국내 선행연구의 연구 유형은 단일대상으로 한 양적연구가 가장 많은 것으로 나타났다(15편, $62.5 \%)$. 진행장소는 연구자 중심의 장소 가 10 편(41.67\%)이며, 진행자에 대한 분석 결과는 직접 개입한 연구가 10편(41.67\%)으로 가장 많은 것으로 나타났다. 또한 프 로그램의 설계를 살펴본 결과, 진행 기간은 4주부터 1년까지 다 양하며 평균 11 주로 나타났으며, 회기 수는 최소 5 회기부터 최 대 96회기로 평균 19회기로 나타났다. 유지기간을 게재한 논문 은 2편(Kim \& Park, 2013; Lee et al, 2016)으로 각각 2주와 7개 월로 나타난 것으로 보아 진행기간과 회기 수를 중점으로 연구 기간이 설계되었다. 종속변인은 어음인지도에 관한 연구가 8편, 의사소통 및 어휘력 증진 6 편, 듣기 및 청각기억력 2편 순으로 나타나며 언어이해력 향상을 통한 의사소통발달을 지원하는 점 이 현재 청능훈련 프로그램 연구들의 특징이라고 볼 수 있다.

셋째, 청각장애 대상 인지 향상에 효과적인 프로그램 제공을 위한 프로그램 결과는 청각적 듣기능력 증진, 소음하 어음인지 력 또는 단어, 문장 인지력 증진, 주관적 만족도 조사에서 불만 항목 감소 및 긍정항목이 향상되었고, 청각 기억력 증진, 의사소 통 및 어휘력이 증진된 것으로 관찰되었다. 중재방법의 경우 전 통적인 접근법을 통해 청능훈련 프로그램을 진행한 연구가 10 편(41.67\%), 직접적인 개입 없이 어플 또는 기기를 통해 중재 한 연구가 3편(12.5\%), 말 산출 및 의사소통 회복 전략을 통한 중재가 3편(12.5\%)으로 나타났다. 이러한 결과는 청능훈련 프로
그램의 중재방법이 직접 개인별로 실시하는 훈련 프로그램이 대 부분임을 보여준다. 향후 각종 디지털기기 및 인터넷을 활용한 간접적인 중재프로그램을 활용한 다양한 방법이 적용되어야 할 것이다.

또한 본 연구의 기준에 따라 분석된 논문들에 따르면 청능훈 련 프로그램 실시 후 일반화가 이루어진 것은 8편(33.33\%)에 불과하다. 이러한 결과는 청각장애아동들이 보장구 착용으로 인한 듣기능력 개선의 효과를 보는 것으로 대부분 만족하기 때 문인 것으로 사료된다. 그러나 청능훈련 프로그램을 통해 향상 된 듣기기술뿐만 아니라 말소리 이해도, 보장구에 대한 만족도 가 삶의 질에 많은 영향을 미친다. 특히 지속적인 청능훈련 프 로그램을 통한 정보상담은 훈련자들의 이해와 수용하는 효용 도를 더욱 높일 수 있다. 따라서 향후 청능훈련 프로그램 설계 시 추후 검사, 유지가 포함되어야 할 필요가 있다.

더불어 본 연구의 분석 논문은 대다수 사례연구이고, 통계 분석을 한 논문은 제한적이었다. 따라서 훈련대상자들은 청력 의 정도가 상이하고 이를 정확하게 정량화하여 나타내기 어렵 기 때문에 연구 내 신뢰도를 확보하는 데 어려움이 따르고, 연 구 간 이질성이 매우 높아 일반적인 결론을 도출하는 데 모호 한 점이 있다. 또한 이러한 제한점을 보완하기 위해 국외 학술 지 등의 범위로 확장하여 다양한 사례를 포함하고, 더욱 엄격 한 기준을 적용하여 분석하는 연구가 진행되어야 할 것이다.

중심 단어 : 청능훈련 프로그램·연구의 질·체계적 문헌고찰. 


\section{Ethical Statement \\ N/A}

\section{Acknowledgments \\ N/A}

\section{Declaration of Conflicting Interests}

There are no conflict of interests.

\section{Funding}

This work was supported by the Soonchunhyang University of Korea (2021).

\section{Author Contributions}

Conceptualization: Joo Hong Kim, Sang Hee Lee. Data curation: Eun Young Cho. Formal analysis: Joo Hong Kim, Sang Hee Lee. Funding acquisition: Joo Hong Kim, Sang Hee Lee. Investigation: all authors. Methodology: Joo Hong Kim, Sang Hee Lee. Project administration: Sang Hee Lee. Resources: Joo Hong Kim, Sang Hee Lee. Software: Joo Hong Kim. Supervision: Sang Hee Lee, Eun Young Cho. Validation: Sang Hee Lee, Eun Young Cho. Visualization: Sang Hee Lee, Eun Young Cho. Writingoriginal draft: Joo Hong Kim, Sang Hee Lee. Writing_review \& editing: Joo Hong Kim, Sang Hee Lee. Approval of final manuscript: all authors.

\section{ORCID iDs}

Joo Hong Kim

https://orcid.org/0000-0003-4630-8767

Sang Hee Lee

\section{REFERENCES}

American Speech-Language-Hearing Association (ASHA). (1984). Definitions of and competencies for aural rehabilitation. ASHA, 26(5), 37-41.

American Speech-Language-Hearing Association (ASHA). (1996). Scope of practice in audiology. ASHA Suppl, 38(2 Suppl 16), 12-15.

Byun, S. W., Kim, S. H., \& Park, S. H. (2017). A meta-analysis on the effectiveness of interventions applied to teens in multicultural families. Journal of Digital Convergence, 15(1), 277-285.

Cho, E. Y. \& Lee, S. D. (2019). A meta-analysis of the program for children of multicultural families. Journal of Learner-Centered Curriculum and Instruction, 19(21), 407-425.

Cho, S. L., Oh S. H., \& Bahng, J. (2018). A case study of auditory training for an adult with sudden sensorineural hearing loss. Audiology and Speech Research, 14(1), 59-64.

Choi, E. A. \& Seong, C. J. (2017). Efficiency of the computer based listening training program for the severe to profound hearing-impaired teenagers and adults. Linguistics [Eoneohag], 0(77), 25-50.

Choi, E. M. \& Park, M. H. (2019). The effects of mind-reading intervention on the improvement of Conversation skills of children with hearing disability. The Korean Study of Education for Hearing-Language Impairments, 10(2), 51-67.

Choi, W. J., Oh S. H., \& Bahng, J. (2017). Efficacy of music training on speech recognition and working memory in children wearing cochlear implants. Audiology and Speech Research, 13(1), 70-77.

Cooper, H. (2010). Research Synthesis and Meta-analysis: A Step-by-Step Approach. (4th ed.). California, CA: SAGE Publication Inc.

Gordon-Salant, S. (2005). Hearing loss and aging: new research findings and clinical implications. Journal of Rehabilitation Research and Development, 42(4 Suppl 2), 9-24.

Hong, H. N. (2013). Case study of auditory training for the acquired hearing loss adult with cochlear implant. Journal of Rehabilitation Research, 17(4), 371-382.

Hwang H. J. \& Seok D. I. (2007). The effect of barrier game reinforcing difference strategy on referential communication skill of children with hear-

ing impairment. Journal of Speech-Language and Hearing Disorders, 16(4), 19-33.

Jo, Y. Y., Bahng, J., \& Lee, J. H. (2013). Case study of auditory training for an elderly hearing aid user. Audiology and Speech Research, 9(2), 190-194.

Jung, P. G. \& Choi, N. R. (2019). The effect of phonics instruction for English underachiever in elementary school: A meta - Analysis. Journal of Learner-Centered Curriculum and Instruction, 19(1), 623-649.

Kim, D. E., Kim, H., Hyun, J. H., Lee, H. J., Sung, H. H., Bae, S. Y., et al. (2018). Interventions using technologies for older adults in long-term care facilities: A systematic review. Journal of Korean Academy of Community Health Nursing, 29(2), 170-183.

Kim, J. H. \& Lee, K. W. (2017). Effects on word and sentence recognition by auditory training using environmental sound for elderly hearing impaired. Audiology and Speech Research, 13(2), 115-122.

Kim, N. K. \& Bahng, J. (2017). Development of a story based auditory training tool and evaluation of the training efficacy for adult hearing impaired listeners. Audiology and Speech Research, 13(2), 133-140.

Kim, S. O. \& Lee, K. W. (2018). Auditory training in an elderly hearing aid user using environmental sounds, stories and questions: A case report. Audiology and Speech Research, 14(3), 204-209.

Kim, S. Y. \& Park, B. D. (2013). The effects of teaching vocabulary on vocabulary competence of university students with hearing impairments and deaf. The Journal of Special Children Education, 15(4), 245-263.

Kim, Y. M. \& Seok, D. I. (1998). The effect of language rehabilitation auditory comprehension program on auditory comprehension ability of hearing disordered students. Journal of Speech and Hearing Disorders, 7(2), $117-129$.

Lee. D. (2018). Verifying the effectiveness of middle-class chorus program for articulation and pitch replaying ability of the students with hearing impairment. The Study of Education for Hearing-Language Impairments, 9(2), 1-23.

Lee, J. H., Lee S. G., \& Bahng, J. (2016). Case study of auditory training for long-term users of hearing aids with poor word recognition. Audiology and Speech Research, 12(3), 190-194.

Lee, J. M. \& Jeon, K. N. (2013). The effects of multicultural education programs on Korean children's multicultural competences: A meta-analytic synthesis. Korean Journal of Teacher Education, 29(2), 279-306.

Lee, M. J. \& Bahng, J. (2020). Efficacy of auditory training using video clips for older adults who wear hearing aids. Audiology and Speech Research, 16(3), 206-216.

Lee, M. S. \& Cho, S. J. (2011). A case report on aural rehabilitation after cochlear implantation in a child with LEOPARD syndrome. The Journal of Special Children Education, 13(3), 357-370.

Lee, S. H. (2005). A study of phonological processes and rehabilitation program for children with cochlear implants. Journal of Speech and Hearing Disorders, 14(3), 43-58.

Lee, S. H. \& Choi, W. J. (2012). Systematic review for new health technology assessment. Journal of the Korean Medical Association, 55(3), 279-291.

Lee, S. O. \& Choi, S. B. (2017). The effects of a phonological awareness intervention on phonological awareness ability and improvement of articulation of infants with hearing impairments. The Journal of Special Children Education, 19(4), 69-87.

Lee, Y. M. \& Lee S. G. (2017). Effect of smartphone applications for aural habilitation in cochlear implanted children: Improvement for auditory memory skills. Audiology and Speech Research, 13(2), 176-188.

Lee, Y. W., Hwang, H. K., \& Lee, J. H. (2019). Efficacy of auditory training on speech recognition in children wearing hearing aids. Audiology and Speech Research, 15(4), 249-257.

Mathers, C., Fat, D. M., \& Boerma, J. T. (2008). The Global Burden of Disease: 2004 Update. Geneva: World Health Organization.

Moher, D., Liberati, A., Tetzlaff, J., Altman, D. G., \& PRISMA Group. (2009). Preferred reporting items for systematic reviews and meta-analyses: the PRISMA statement. Annals of Internal Medicine, 151(4), 264-269, W64.

Park, S. A., Shin, H. J., \& Park, E. S. (2019). The effect of nonsense two-syl- 
lable minimal contrast pair auditory training on school-aged cochlear implant wearers improvement in final plosive consonant auditory perception. Journal of Speech-Language and Hearing Disorders, 28(1), 5969.

Park, S. H. \& Han, W. (2019). A systematic review for auditory training effect based on Korean database. Audiology and Speech Research, 15(3), 145-159.

Seo, K. W. \& Moon, H. K. (2017). Method logical quality of meta-analyses on the multi-culture. Asia-Pacific Journal of Multimedia Services Convergent with Art, Humanities, and Sociology, 7(8), 211-223.

Shea, B. J., Grimshaw J. M., Wells, G. A., Boers, M., Andersson, N., Hamel, C., et al. (2007). Development of AMSTAR: A measurement tool to assess the methodological quality of systematic reviews. BMC Medical Research Methodology, 7:10.

Sweetow, R. W. \& Sabes, J. H. (2006). The need for and development of an adaptive Listening and Communication Enhancement $\left(\mathrm{LACE}^{\mathrm{TM}}\right)$ Program. Journal of the American Academy of Audiology, 17(8), 538-558.
Tark, P. K., Kim, S. H., Park, S. I., Lee, S. B., Bae, H. W., \& Park, J. H. (2019). Effect of auditory training with music for hearing aid users. Audiology and Speech Research, 15(3), 205-213.

Yeo, S. H., Bahng, J., \& Lee, J. H. (2014). Efficacy of auditory training using sentences in noise for hearing aid users. Audiology and Speech Research, 10(1), 65-75.

Yoon, H. B. \& Hwang, D. S. (2005). A review study for development of auditory training program to hearing-impaired children. The Journal of Special Children Education, 7(3), 269-289.

Yoon, J. E., Kim, J. S., \& Park, H. J. (2016). Early auditory rehabilitation of an infant with unilateral profound hearing loss: A case study. Audiology and Speech Research, 12(2), 115-125.

Yu, J. H., Chun, H. J., Song, C. G., \& Han, W. (2014). Case study of speech perception enhancement in hearing-impaired adult by auditory training program of mobile device. Audiology and Speech Research, 10(2), 158-168. 


\section{APPENDIX}

\section{Study List for the Systematic Review}

Cho, S. L., Oh, S. H., \& Bahng, J. (2018). A case study of auditory training for an adult with sudden sensorineural hearing loss. Audiology and Speech Research, 14(1), 59-64.

Choi, E. A. \& Seong, C. J. (2017). Efficiency of the computer based listening training program for the severe to profound hearing-impaired teenagers and adults. Linguistics [Eoneohag], 0(77), 25-50.

Choi, E. M. \& Park, M. H. (2019). The effects of mind-reading intervention on the improvement of Conversation skills of children with hearing disability. The Korean Study of Education for Hearing-Language Impairments, 10(2), 51-67.

Choi, W. J., Oh S. H., \& Bahng, J. (2017). Efficacy of music training on speech recognition and working memory in children wearing cochlear implants. Audiology and Speech Research, 13(1), 70-77.

Hong, H. N. (2013). Case study of auditory training for the acquired hearing loss adult with cochlear implant. Journal of Rehabilitation Research, 17(4), 371-382.

Hwang H. J. \& Seok D. I. (2007). The effect of barrier game reinforcing difference strategy on referential communication skill of children with hearing impairment. Journal of Speech-Language and Hearing Disorders, 16(4), $19-33$.

Jo, Y. Y., Bahng, J., \& Lee, J. H. (2013). Case study of auditory training for an elderly hearing aid user. Audiology and Speech Research, 9(2), 190-194.

Kim, J. H. \& Lee, K. W. (2017). Effects on word and sentence recognition by auditory training using environmental sound for elderly hearing impaired. Audiology and Speech Research, 13(2), 115-122.

Kim, N. K. \& Bahng, J. (2017). Development of a story based auditory training tool and evaluation of the training efficacy for adult hearing impaired listeners. Audiology and Speech Research, 13(2), 133-140.

Kim, S. O. \& Lee, K. W. (2018). Auditory training in an elderly hearing aid user using environmental sounds, stories and questions: A case report. Audiology and Speech Research, 14(3), 204-209.

Kim, S. Y. \& Park, B. D. (2013). The effects of teaching vocabulary on vocabulary competence of university students with hearing impairments and deaf. The Journal of Special Children Education, 15(4), 245-263.

Kim, Y. M. \& Seok, D. I. (1998). The effect of language rehabilitation auditory comprehension program on auditory comprehension ability of hearing disordered students. Journal of Speech and Hearing Disorders, 7(2), 117-129.

Lee. D. (2018). Verifying the effectiveness of middle-class chorus program for articulation and pitch replaying ability of the students with hearing impairment. The Study of Education for Hearing-Language Impairments, 9(2), 1-23.

Lee, J. H., Lee S. G., \& Bahng, J. (2016). Case study of auditory training for long-term users of hearing aids with poor word recognition. Audiology and Speech Research, 12(3), 190-194.

Lee, M. J. \& Bahng, J. (2020). Efficacy of auditory training using video clips for older adults who wear hearing aids. Audiology and Speech Research, 16(3), 206-216.

Lee, M. S. \& Cho, S. J. (2011). A case report on aural rehabilitation after cochlear implantation in a child with LEOPARD syndrome. The Journal of Special Children Education, 13(3), 357-370.

Lee, S. O. \& Choi, S. B. (2017). The effects of a phonological awareness intervention on phonological awareness ability and improvement of articulation of infants with hearing impairments. The Journal of Special Children Education, 19(4), 69-87.

Lee, Y. M. \& Lee S. G. (2017). Effect of smartphone applications for aural habilitation in cochlear implanted children: Improvement for auditory memory skills. Audiology and Speech Research, 13(2), 176-188.

Lee, Y. W., Hwang, H. K. \& Lee, J. H. (2019). Efficacy of auditory training on speech recognition in children wearing hearing aids. Audiology and Speech Research, 15(4), 249-257. 
Park, S. A., Shin, H. J., \& Park, E. S. (2019). The effect of nonsense two-syllable minimal contrast pair auditory training on school-aged cochlear implant wearers improvement in final plosive consonant auditory perception. Journal of SpeechLanguage and Hearing Disorders, 28(1), 59-69.

Tark, P. K., Kim, S. H., Park, S. I., Lee, S. B., Bae, H. W. \& Park, J. H. (2019). Effect of auditory training with music for hearing aid users. Audiology and Speech Research, 15(3), 205-213.

Yeo, S. H., Bahng, J., \& Lee, J. H. (2014). Efficacy of auditory training using sentences in noise for hearing aid users. Audiology and Speech Research, 10(1), 65-75.

Yoon, J. E., Kim, J. S., \& Park, H. J. (2016). Early auditory rehabilitation of an infant with unilateral profound hearing loss: A case study. Audiology and Speech Research, 12(2), 115-125.

Yu, J. H., Chun, H. J., Song, C. G., \& Han, W. (2014). Case study of speech perception enhancement in hearing-impaired adult by auditory training program of mobile device. Audiology and Speech Research, 10(2), 158-168. 